\title{
Exploration of Single-Atom X-ray Analysis in an Analytical Electron Microscope
}

M. Watanabe*

* Dept of Materials Science and Engineering, Lehigh University, Bethlehem. PA 18015.

Characterization of individual atoms randomly distributed in materials, which requires atomic-level spatial resolution as well as single-atom detection sensitivity, is one of the ultimate goals in chemical analysis. In fact, some of fundamental materials problems such as phase transformations and interface-related phenomena might be controlled by small composition fluctuations in localized (atomic-level) areas of materials. Thus, the single-atom characterization/analysis is essential for further understanding such phenomena for materials development. The latest aberration-corrected analytical electron microscopes (AEMs) in combination with electron energy-loss spectrometry (EELS) routinely offers single-atom imaging and analysis[e.g. 1]. Recently, Lovejoy et al. have proved that such single-atom analysis is also possible by using X-ray energy-dispersive spectrometry (XEDS) in an aberration-corrected AEM[2]. In addition, the recent advance in large solid angle silicon-drift X-ray detectors (SDDs) improves the poor signal-collection efficiency. Conversely, it is still challenging to perform single atom analysis by XEDS due to the poor analytical sensitivity. Therefore, this paper explores how to achieve single-atom analysis by XEDS.

For single-atom X-ray analysis, evaluation of the analytical sensitivity is essential since spatial resolution already reaches to the atomic scale in the aberration-corrected AEMs. Figure 1 compares X-ray spectra from Fe- $0.15 \mathrm{wt} \% \mathrm{P}$ specimen, simulated with/without Poisson noise by legacy Desktop Spectrum Analyzer (DTSA) software [3]. If the peak intensity above background is higher than the background-noise fluctuation term $3(2 \mathrm{~B})^{1 / 2}$ (B: background intensity), the peak can be detected [4]. Then, the detectability limit in terms of composition, i.e. the minimum mass fraction (MMF), can be determined at various specimen thickness and in certain instrumental conditions. Fig. 2 shows MMF values of $\mathrm{P}$ in Fe estimated as a function of specimen thickness for a conventional Si(li) detector (solid angle: $0.15 \mathrm{sr}$ ) and a large solid-angle SDD (0.6 sr) in a $200 \mathrm{kV}$ instrument. In this simulation, each MMF value was determined by averaging 50 spectra simulated with different random noise conditions. The acquisition time for each spectrum is $100 \mathrm{~s}$. As the specimen thickness increases, the X-ray signal increases, and hence the MMF value is improved. Although the large-angle SDD offers better analytical sensitivity, the MMF in AEM is $\sim 0.1 \mathrm{wt} \%$, which is still much worse than that of bulk sample analysis in electron probe microanalyzers. Furthermore, it is hard to evaluate a number of atoms from the MMF value.

With knowledge of electron-specimen interaction volume (analysed), the MMF values can be converted to the more convenient figure, minimum detectable atoms (MDA) [5]. Estimated analysed volumes of $\mathrm{Fe}$ in conventional and aberration-corrected $200 \mathrm{kV}$ are plotted against specimen thickness in Fig. 3. At very thin regions, the probe size is more dominant than beam broadening. The MDA values can be determined from the analysed volume in combination with the MMF values. Fig. 4 compares MDA values determined for conventional AEM with a conventional $\mathrm{Si}(\mathrm{Li})$ detector, aberration-corrected AEM with a conventional $\mathrm{Si}(\mathrm{Li})$ detector and with a large-angle SDD. Despite of the poor MMF in thinner regions, the MDA value is improved as the specimen thickness decreases. According to these results, only $\sim 20 \mathrm{P}$ atoms are 
detectable by using the conventional AEM under these specific conditions. This value may be improved to a single atom level in the aberration-corrected instruments. The large-angle SDD expands the specimen thickness range for the single atom detection.

\section{References}

[1] S.J. Pennycook \& P.D. Nellist ed. Scanning Transmission Electron Microscopy: Imaging and Analysis, Springer, NY, (2011).

[2] T. Lovejoy et al., Appl.Phys. Lett. 100 (2012), 154101 (4 pages).

[3] C.E. Fiori et al. Public domain DTSA software package (1992).

[4] D.B. Williams \& C.B. Carter. Transmission Electron Microscopy, Springer, NY (2009).

[5] M. Watanabe \& D.B. Williams, Ultramicrosc. 78 (1999) 89

[6] The author wishes to acknowledge financial support from the NSF through grants DMR-0804528 and DMR-1040229.

Fig. 1

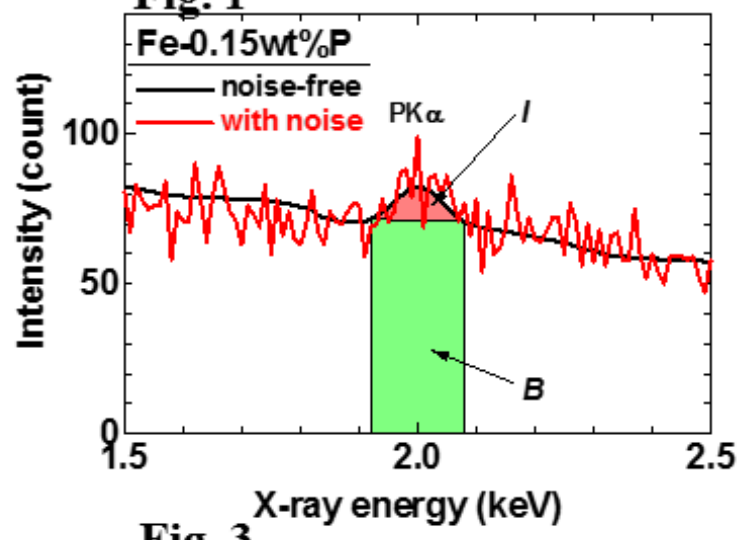

Fig. 3

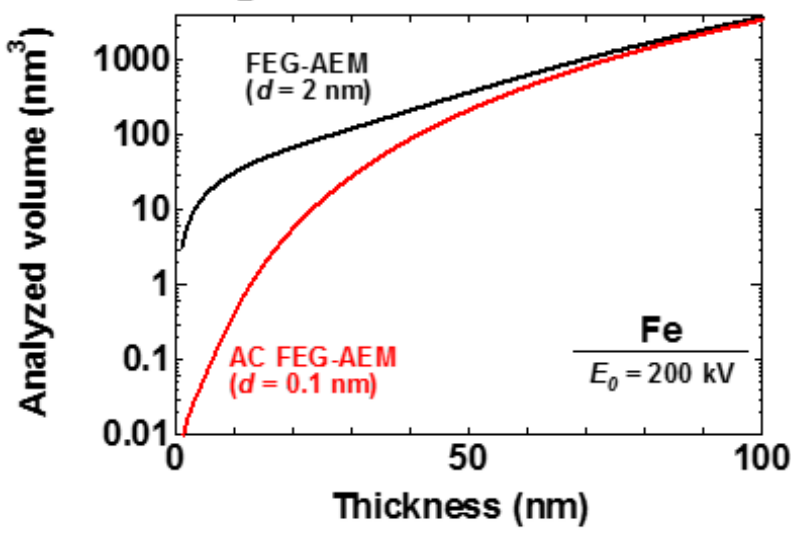

Fig. 2

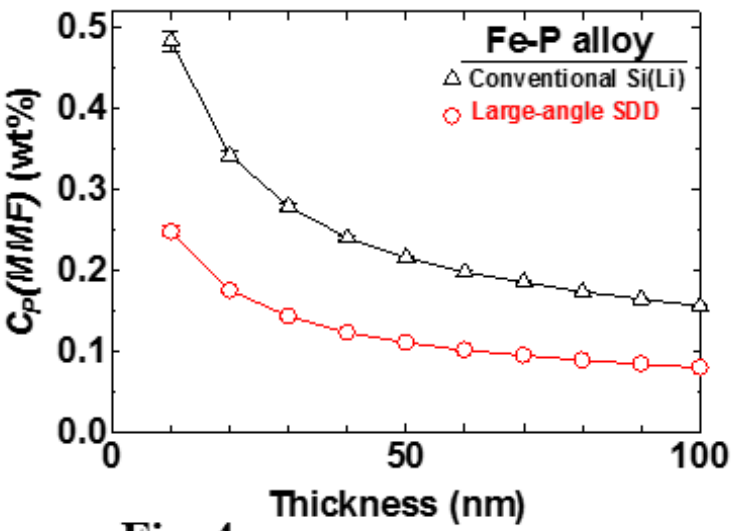

Fig. 4

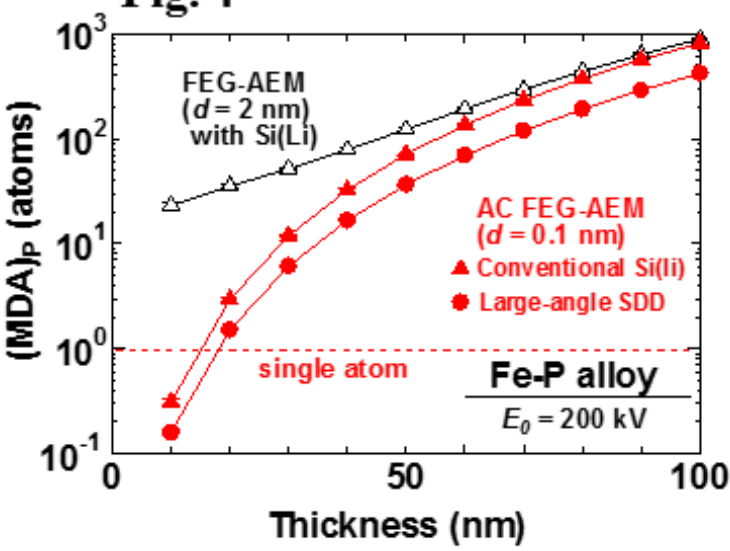

Figure 1: A comparison of two X-ray spectra from a $100-\mathrm{nm}$-thick Fe- $0.15 \mathrm{wt} \% \mathrm{P}$ simulated with and without noise in a $200 \mathrm{kV}$ instrument for $100 \mathrm{~s}$.

Figure 2: The MMF of $\mathrm{P}$ in Fe, determined from simulated $\mathrm{X}$-ray spectra for conventional $\mathrm{Si}(\mathrm{Li})$ and large-angle SDD.

Figure 3: The analyzed volume of Fe determined for conventional AEM and aberration-corrected AEM, plotted against the specimen thickness.

Figure 4: The MDA of $\mathrm{P}$ in Fe calculated from the MMF in Fig.2 with the analyzed volume. 